\title{
Portrait de la compression et de l'expansion de la morbidité au Canada : évolution de l'espérance de vie et de l'espérance de vie ajustée en fonction de la santé, 1994-2010
}

\author{
Colin Steensma, M. Sc. (1,2); Lidia Loukine, M. Sc. (1); Bernard C. K. Choi, Ph. D. (1,3,4)
}

Cet article a fait l'objet d'une évaluation par les pairs.

Diffuser cet article sur Twitter

\begin{abstract}
Résumé
Introduction : Notre étude visait à examiner s'il y a eu une expansion ou une compression de la morbidité au Canada, à l'échelle tant nationale que provinciale, en suivant l'évolution de l'espérance de vie (EV) et de l'espérance de vie ajustée en fonction de la santé (EVAS) entre 1994 et 2010. Une " compression », qui correspond à une diminution de la proportion de la vie marquée par des problèmes de santé, survient lorsque l'EVAS augmente plus rapidement que l'EV. Inversement, une "expansion », qui désigne une augmentation de la proportion de la vie marquée par des problèmes de santé, survient lorsque l'EVAS est stable ou augmente plus lentement que l'EV.
\end{abstract}

Méthodologie : Nous avons estimé l'EV à l'aide des données de Statistique Canada sur la mortalité et sur la population. Nous avons utilisé les données sur la qualité de vie liée à la santé (c.-à-d. morbidité) de l'Enquête nationale sur la santé de la population (1994-1999) et de l'Enquête sur la santé dans les collectivités canadiennes (2000-2010) pour calculer l'EVAS. Nous avons établi des tables de mortalité abrégées pour sept intervalles de temps correspondant aux cycles d'enquête disponibles durant la période de 1994 à 2010, ainsi que pour les deux sexes et les dix provinces canadiennes. Les tendances nationales et provinciales ont été examinées à la naissance, à 20 ans et à 65 ans.

Résultats : Dans l'ensemble, nous avons constaté une hausse annuelle moyenne statistiquement significative de l'EVAS chez les deux sexes aux trois âges évalués, sauf chez les nouveau-nés de sexe féminin. L'EVAS à la naissance a augmenté en moyenne par année, entre 1994 et 2010 , de $0,2 \%(p=0,08)$ chez les sujets de sexe féminin et de $0,3 \%$ $(p<0,001)$ chez ceux de sexe masculin. À l'échelle nationale, les trois groupes d'âge ont présenté une augmentation annuelle moyenne non statistiquement significative de la proportion de la vie marquée par des problèmes de santé, sauf les hommes de 65 ans, qui ont présenté une diminution non significative. À l'échelle provinciale, nous avons observé une augmentation significative de la proportion de la vie marquée par des problèmes de santé à Terre-Neuve-et-Labrador et à l'Île-du-Prince-Édouard.

Conclusion : Notre étude n’a fait ressortir aucune tendance générale nette indiquant une compression ou une expansion de la morbidité à l'échelle nationale au Canada entre 1994 et 2010. Nos résultats indiquent qu'il y a eu une expansion de la morbidité à Terre-Neuve-et-Labrador et à l'Île-du-Prince-Édouard. L'étude a mis en évidence l'importance de continuer à suivre les tendances à long terme concernant l'EV et l'EVAS pour vérifier la présence d'une compression ou d'une expansion de la morbidité. D'autres études devraient être entreprises pour éclaircir les causes de l'expansion de la morbidité observée à Terre-Neuve-et-Labrador et à l'Île-du-Prince-Édouard.

\section{Points saillants}

- L'espérance de vie (EV) et l'espérance de vie ajustée en fonction de la santé (EVAS) ont augmenté de façon constante de 1994 à 2010 chez les Canadiens des deux sexes.

- Les gains d'EVAS observés dans la population canadienne durant la période à l'étude étaient principalement associés à la diminution de la mortalité.

- L'espérance de santé de la population canadienne semble avoir été relativement stable de 1994 à 2010 : aucune tendance globale nette vers une compression ou une expansion de la morbidité n'est ressortie au Canada durant la période à l'étude.

Mots-clés : espérance de vie, espérance de santé, compression, expansion, mortalité, morbidité, tendance, qualité de vie liée à la santé

\section{Introduction}

Deux grands scénarios potentiels ont été proposés pour l'évolution de la mortalité et de la morbidité. Dans le premier, la « compression de la morbidité », on assiste à une hausse de l'espérance de vie (EV) et à une diminution de la proportion de la vie marquée par des problèmes de santé ou des incapacités graves ${ }^{1-3}$. Ce scénario peut se

Rattachement des auteurs :

1. Agence de la santé publique du Canada, gouvernement du Canada, Ottawa (Ontario), Canada

2. Département de médecine sociale et préventive, Université de Montréal, Montréal (Québec), Canada

3. Dalla Lana School of Public Health, University of Toronto, Toronto (Ontario), Canada

4. Centre de recherche sur la prévention des blessures, Collège médical de l'Université de Shantou, Shantou, Chine

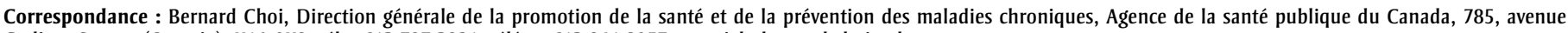
Carling, Ottawa (Ontario), K1A 0K9; tél. : 613-797-3821; téléc. : 613-941-2057; courriel : bernard.choi@phac-aspc.gc.ca 
réaliser lorsque des changements dans les tendances des maladies retardent l'apparition de celles-ci. Les personnes vivent plus longtemps (diminution de la mortalité) et en meilleure santé (diminution de la morbidité).

Dans le second scénario, l'« expansion de la morbidité », l'espérance de vie augmente, mais la proportion de la vie marquée par des problèmes de santé ou des incapacités augmente aussi ${ }^{4-5}$. Ce scénario se produit lorsque des percées médicales permettent de diminuer la mortalité et le taux de létalité des maladies, sans toutefois modifier leurs tendances. L'amélioration des soins de santé favorise la survie des personnes, qui restent en vie malgré leur maladie. Les personnes vivent donc plus longtemps (diminution de la mortalité), mais elles sont aussi atteintes de problèmes de santé plus longtemps (augmentation de la morbidité). Notre étude, qui vise à déterminer lequel de ces scénarios est dominant, va fournir des renseignements importants sur le fardeau global de la maladie pour les responsables de la santé ainsi que sur les conséquences pour la planification stratégique des services de soins de santé et de leur prestation.

L’espérance de santé, définie comme l'espérance de vie dans un état de santé défini $^{6,7}$, est un outil important dans la surveillance de l'évolution de la santé de la population et dans l'évaluation des données sur la compression et l'expansion de la morbidité. L'espérance de vie ajustée en fonction de la santé (EVAS), définie comme " le nombre moyen d'années de vie en bonne santé que devrait connaître une personne, compte tenu de la mortalité et de la morbidité courantes au moment où est établie la mesure $»^{8}$, est un paramètre utile pour mesurer l'espérance de santé.

Les études sur les tendances nationales récentes en matière d'espérance de santé dans les pays économiquement développés $^{9-19}$ ont fait ressortir des données en faveur des deux scénarios : certains pays et régions autonomes semblent présenter une expansion de la morbidité $e^{-12}$, tandis que d'autres présentent une compression de la morbidité13-16. Dans un cas, les scénarios varient selon la tranche d'âge ${ }^{17}$. Au Canada, les études sur l'évolution de l'espérance de santé à l'échelle nationale ont donné des résultats contradictoires. Une étude a conclu qu'il y a eu une période d'expansion de la morbidité de 1986 à 1991 suivie d'une compression de la morbidité de 1991 à $1996^{18}$, alors qu'une comparaison de l'espérance de santé en 1994 et en 2007 a laissé entendre qu'il y avait eu, dans l'ensemble, une expansion de la morbidité entre les deux années à l'étude ${ }^{19}$.

L'une des difficultés liées à l'évaluation de l'évolution de l'espérance de santé d'après les enquêtes nationales disponibles est le manque de données uniformes au fil du temps. Un grand nombre d'études sur ce sujet ont utilisé des mesures différentes de l'incapacité durant les périodes de déclaration ${ }^{12,15,16}$ ou encore un nombre limité de périodes de déclaration ${ }^{11,16,17,19}$.

De plus, l'évolution de l'espérance de santé a été peu évaluée à l'échelle infranationale ${ }^{20-22}$. À l'échelle provinciale au Canada, une étude menée au Québec ${ }^{22}$ a révélé que la proportion de la vie vécue avec une incapacité était plus grand en 1998 qu'en 1986, indiquant une expansion de la morbidité durant cet intervalle. Au Canada, les estimations provinciales de l'évolution de l'espérance de santé constituent un point de départ utile pour les décideurs de la santé publique lors de l'allocation des ressources, car c'est à l'échelle provinciale que les soins de santé sont mis en œuvre.

Notre étude vise à évaluer l'évolution de l'espérance de vie ajustée en fonction de la santé (EVAS) dans la population canadienne entre 1994 et 2010 à l'aide de plusieurs cycles d'enquêtes nationales représentatives semblables, reposant sur la même mesure de la santé fonctionnelle (Indice de l'état de santé, ou IES). Plus spécifiquement, nous avons examiné les tendances en matière d'expansion ou de compression de la morbidité en mesurant l'évolution de la proportion de la vie marquée par des problèmes de santé. Ces tendances ont été examinées à la naissance, à 20 ans et à 65 ans, par sexe et par province.

\section{Méthodologie}

Nous avons utilisé des données de Statistique Canada sur la mortalité et sur la population pour estimer l'espérance de vie durant sept intervalles de temps couvrant la période de 1994 à 2010. Ces intervalles correspondaient aux années pour lesquelles il existait des données d'enquête permettant de calculer l'EVAS (voir plus loin). Les valeurs de l'EVAS sont aussi fondées sur des données autodéclarées supplémentaires concernant la morbidité tirées de
l'Indice de l'état de santé (IES) de l'Enquête nationale sur la santé de la population (ENSP) ainsi que de l'Enquête sur la santé dans les collectivités canadiennes (ESCC) menées par Statistique Canada.

L'ENSP et l'ESCC ont été conçues pour collecter des renseignements sur la santé de la population canadienne vivant dans un ménage. Durant les trois premiers cycles (1994-1995, 1996-1997 et 1998-1999), l'ENSP comportait un volet transversal et un volet longitudinal. À compter de 2000, le volet transversal de l'ENSP a été transféré à l'ESCC. Par conséquent, les données transversales pour 1994 à 1999 proviennent de l'ENSP et celles pour 2000 à 2010, de l'ESCC.

Le premier cycle de collecte de données de l'ENSP s'est déroulé en 1994-1995. Les cycles subséquents ont eu lieu aux deux ans. L'échantillon de base initial était constitué de 17276 personnes, à l'échelle des dix provinces canadiennes ${ }^{23}$. Le taux de réponse des ménages au volet transversal à l'échelle nationale a été pour les trois premiers cycles de respectivement 88,7 \%, $82,6 \%$ et $89,7 \%$.

L'ESCC, qui a porté sur un échantillon d'environ 130000 répondants, visait à fournir des estimations fiables à l'échelle des régions sanitaires locales ${ }^{24}$. Nous avons utilisé les cycles de données de l'ESCC pour lesquels l'IES avait été établi à l'égard de toutes les provinces canadiennes : 2000-2001, 2003, 2005 et 2009-2010. Le taux de réponse des ménages durant ces cycles a varié entre $72,3 \%$ (2009-2010) et 84,7 \% (2000-2001). Les données des cycles 2003 et 2005 de l'ESCC ont été collectées sur une année.

L'ENSP visait des personnes de tous âges, alors que l'ESCC a exclu les enfants de moins de 12 ans. Nous avons donc établi une valeur de 0,99 pour l'IES des moins de 12 ans en supposant que les enfants de cette tranche d'âge n'étaient pas tous en parfaite santé. Nous avons choisi la valeur de 0,99 , car la qualité de vie liée à la santé est normalement très élevée chez les enfants de moins de 12 ans. Les deux enquêtes ont exclu les personnes vivant sur les réserves indiennes et les terres de la Couronne, les résidents des établissements de soins de santé, les membres à temps plein des Forces canadiennes vivant sur des bases des Forces canadiennes, ainsi que les habitants de certaines régions éloignées de l'Ontario et du Québec. Nous 
avons exclu les trois territoires canadiens de l'analyse, car il manquait de données à leur sujet dans certains des cycles retenus.

Nous avons utilisé des données de Statistique Canada ${ }^{25}$ pour estimer les taux de mortalité par province, par sexe et par tranche d'âge de cinq ans. À l'aide de la méthode de la moyenne mobile sur trois ans, nous avons établi des estimations solides, ce qui est particulièrement important pour le calcul des valeurs concernant les provinces ayant une faible population. Il faut des données sur trois ans pour arriver à un volume suffisant pour assurer la fiabilité des taux de mortalité utilisés pour les estimations de l'EV et de l'EVAS. Les intervalles de trois ans que nous avons utilisés correspondent aux sept cycles d'enquête ayant servi à calculer l'EVAS : 1993 à 1995, 1995 à 1997, 1997 à 1999, 2000 à 2002, 2002 à 2004, 2004 à 2006 et 2009 à 2011.

Nous avons mesuré la qualité de vie liée à la santé (QVLS) à l'aide de l'Indice de l'état de santé Health Utilities Index Mark 3 (IES3), qui est disponible pour les deux enquêtes ${ }^{24,26}$. L'IES est un instrument de mesure qui permet de classer les états de santé en fonction de huit attributs (vue, ouïe, parole, mobilité, dextérité, émotion, cognition et douleur). Chacun des attributs comporte cinq ou six échelons, qui vont d'un fonctionnement normal à un fonctionnement gravement limité. Les valeurs de l'indice varient entre - 0,36 (pire état de santé possible, c'est-à-dire un état auquel la mort serait préférable) à 1,0 (meilleur état de santé possible) en passant par 0,0 (mort). Les différences de 0,03 et plus au score total de l'IES sont considérées comme cliniquement importantes ${ }^{26}$.

Nous avons combiné la méthode de Chiang $^{27}$ et la méthode de $\mathrm{Hsieh}^{28}$ pour produire des tables de mortalité par sexe et par tranche d'âge standard (moins de 1 an, $1-4,5-9, \ldots, 80-85$ et 85 ans et plus). La méthode de Chiang est une méthode éprouvée, largement utilisée partout dans le monde pour établir des tables de mortalité abrégées. Elle consiste à utiliser des taux de mortalité selon l'âge pour calculer la probabilité de mourir à chaque intervalle d'âge et à appliquer ce résultat à une cohorte de population hypothétique. La méthode de Hsieh, qui est fondée sur la fonction de Gompertz, a servi à produire une estimation précise de l'EV dans la tranche d'âge la plus élevée ( 85 ans et plus). Nous avons utilisé une méthode de
Sullivan modifiée ${ }^{7}$ pour calculer l'EVAS. Selon cette méthode, les " années de vie vécues " dans chaque intervalle d'âge sont ajustées en fonction de l'IES. La variance de l'EVAS a été calculée à l'aide de la méthode de Mathers ${ }^{9}$, qui prend en compte la variance de la probabilité de mourir fondée sur les tables de mortalité et la variance de l'IES.

Pour analyser l'évolution de l'espérance de santé, plus spécifiquement les scénarios de morbidité, nous avons calculé le pourcentage annuel de variation de l'EVAS ainsi que la " proportion de la vie marquée par des problèmes de santé », soit (EV-EVAS)/EV, en appliquant un modèle de régression log-linéaire. Il est nécessaire d'évaluer la différence entre l'EVAS et l'EV par rapport à l'EV car, comme l'EVAS est essentiellement une mesure de l'EV pondérée en fonction de la qualité de vie liée à la santé, elle est fortement corrélée à l'EV.

Pour mesurer le rôle de la QVLS dans l'évolution de l'EVAS au Canada, nous avons réparti les différences sur le plan de l'EVAS entre les composantes mortalité et par rapport à la période de déclaration initiale (1994-1995), à l'aide d'une méthode d'Arriaga $^{29}$ adoptée pour la méthode de Sullivan $^{30}$.

\section{Résultats}

Les valeurs moyennes de l'IES ont fluctué durant la période à l'étude au sein de la morbidité (QVLS), pour toutes les périodes

population canadienne de 12 ans et plus (figure 1). Nous avons observé pour les deux sexes une augmentation moyenne statistiquement significative de l'IES tout juste inférieure à 0,01 : de 0,862 en 1994-1995 à 0,871 en 2009-2010 chez les femmes $(p<0,01)$, et de 0,879 en 1994-1995 à 0,886 en 2009-2010 chez les hommes $(p<0,05)$. Cependant, bien qu'une hausse cliniquement importante de la QVLS ait été observée pour les deux sexes entre 1994-1995 et 1996-1998 (c.-à-d. augmentation de l'IES de 0,03 ou plus), la hausse générale de la QVLS sur l'ensemble de la période à l'étude ne s'est pas révélée cliniquement importante pour l'un ou l'autre.

L'EVAS a augmenté de façon constante chez les Canadiens des deux sexes durant la période de déclaration (tableaux 1A, 1B et 1C). Chez les femmes, l'EVAS a augmenté de 3,3 ans à la naissance, de 3,1 ans à 20 ans et de 2,0 ans à 65 ans, mais seules les femmes à 20 et à 65 ans ont présenté des augmentations annuelles moyennes significatives de l'EVAS : respectivement $0,2 \%(p<0,05)$ et $0,7 \%(p<0,001)$. Chez les hommes, l'EVAS a augmenté, entre 19941995 et 2009-2010, de 4,3 ans à la naissance, de 4,0 ans à 20 ans et de 2,6 ans à 65 ans. Ces résultats correspondent à des augmentations annuelles moyennes significatives de l'EVAS pour chacun des groupes d'âge : $0,3 \%$ à la naissance, $0,4 \%$ à 20 ans et $1,2 \%$ à 65 ans ( $p<0,001$ dans les trois cas).

La proportion de la vie marquée par des problèmes de santé, soit (EV-EVAS)/EV, a

FIGURE 1

Valeurs moyennes de l'Indice de l'état de santé, population de 12 ans et plus, par sexe et par période de déclaration, Canada, 1994-2010

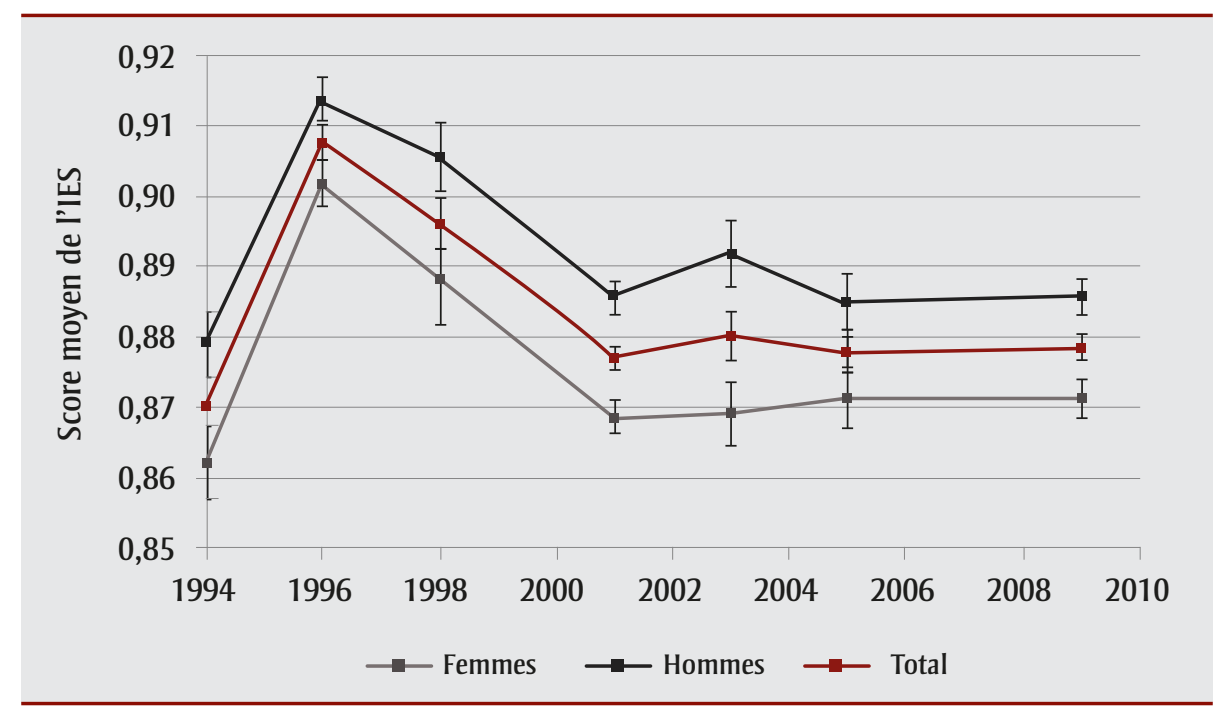

Abréviation : IES, Indice de l'état de santé. 
TABLEAU 1A

Espérance de vie, espérance de vie ajustée en fonction de la santé et proportion de la vie marquée par des problèmes de santé à la naissance, par sexe et par période de déclaration, Canada, 1994-2010

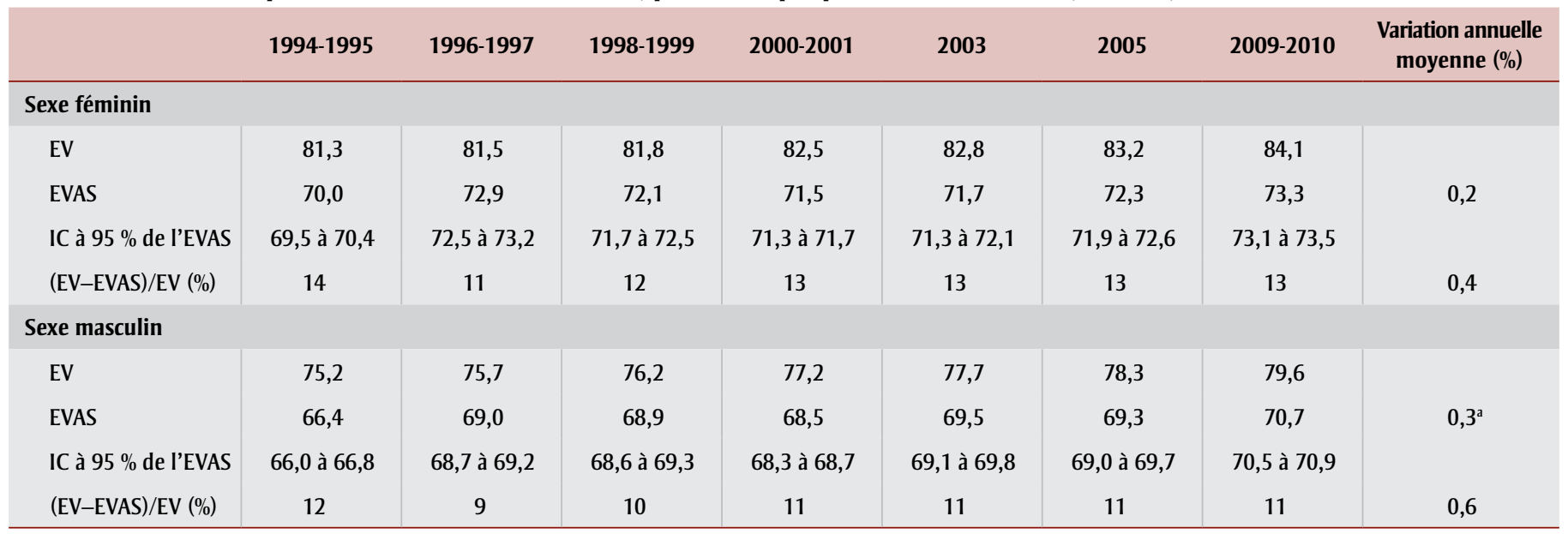

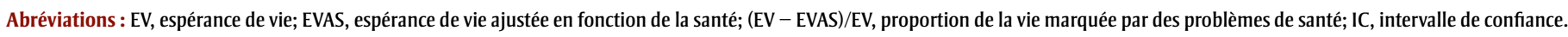
${ }^{\text {a }} p<0,05$.

fluctué pendant la période à l'étude. Chez les nouveau-nés canadiens, elle a diminué entre 1994-1995 (14\% chez les filles et $12 \%$ chez les garçons) et 1996-1997 (11 \% chez les filles et $9 \%$ chez les garçons), ce qui traduit une compression de la morbidité. Elle a ensuite augmenté jusqu'en 2009-2010 (13 \% chez les filles et $11 \%$ chez les garçons) (tableau $1 \mathrm{~A}$ ), ce qui indique une expansion de la morbidité. Sur l'ensemble de la période à l'étude, elle n'a pas changé de façon significative (tableaux $1 \mathrm{~A}, 1 \mathrm{~B}$ et $1 \mathrm{C})$, ce qui ne correspond ni à une compression ni à une expansion de la morbidité. Cette tendance a aussi été observée chez les hommes et les femmes à 20 ans et chez les femmes à 65 ans (tableaux $1 \mathrm{~B}$ et $1 \mathrm{C}$ ). Les hommes à 65 ans ont présenté une diminution annuelle moyenne non significative de la proportion de la vie marquée par des problèmes de santé.

La répartition des différences sur le plan de l'EVAS à la naissance selon la période de déclaration par rapport aux valeurs initiales de 1994-1995 a fait ressortir des gains constants de l'EVAS associés à la composante mortalité (tableau 2). Les gains attribués à la QVLS (composante morbidité) ont quant à eux varié tout au long de la période à l'étude, ce qui indique l'absence de tendance claire vers une expansion ou une compression de la morbidité. L'évolution de la situation est similaire pour les deux sexes.

Si l'évolution de l'EVAS dans les provinces correspond globalement à celle observée à l'échelle nationale (tableaux $3 \mathrm{~A}$ et $3 \mathrm{~B}$ ), quelques différences sont néanmoins perceptibles. Entre 1994-1995 et 2009-2010, des augmentations annuelles moyennes significatives de l'EVAS à la naissance (les deux sexes confondus) ont été observées seulement en Colombie-Britannique et au Québec.

TABLEAU 1B

Espérance de vie, espérance de vie ajustée en fonction de la santé et proportion de la vie marquée par des problèmes de santé à 20 ans, par sexe et par période de déclaration, Canada, 1994-2010

\begin{tabular}{|c|c|c|c|c|c|c|c|c|}
\hline & 1994-1995 & 1996-1997 & 1998-1999 & $2000-2001$ & 2003 & 2005 & 2009-2010 & $\begin{array}{c}\text { Variation annuelle } \\
\text { moyenne (\%) }\end{array}$ \\
\hline \multicolumn{9}{|l|}{ Sexe féminin } \\
\hline EVAS & 51,4 & 54,0 & 53,1 & 52,7 & 53,1 & 53,5 & 54,5 & $0,2^{\mathrm{a}}$ \\
\hline IC à $95 \%$ de l'EVAS & 51,0 à 51,9 & 53,7 à 54,3 & 52,7 à 53,5 & 52,5 à 52,9 & 52,7 à 53,5 & 53,1 à 53,9 & 54,3 à 54,8 & \\
\hline \multicolumn{9}{|l|}{ Sexe masculin } \\
\hline EV & 55,7 & 56,2 & 56,7 & 57,7 & 58,1 & 58,7 & 59,9 & \\
\hline EVAS & 48,0 & 50,1 & 50,1 & 49,8 & 50,9 & 50,8 & 52,0 & $0,4^{\mathrm{a}}$ \\
\hline IC à 95 \% de l'EVAS & 47,6 à 48,3 & 49,8 à 50,3 & 49,7 à 50,4 & 49,6 à 50,0 & 50,5 à 51,2 & 50,4 à 51,2 & 51,8 à 52,2 & \\
\hline (EV-EVAS)/EV (\%) & 14 & 11 & 12 & 14 & 12 & 13 & 13 & 0,5 \\
\hline
\end{tabular}

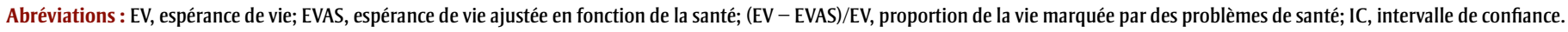
${ }^{\text {a }} p<0,05$. 
TABLEAU 1C

Espérance de vie, espérance de vie ajustée en fonction de la santé et proportion de la vie marquée par des problèmes de santé à 65 ans, par sexe et par période de déclaration, Canada, 1994-2010

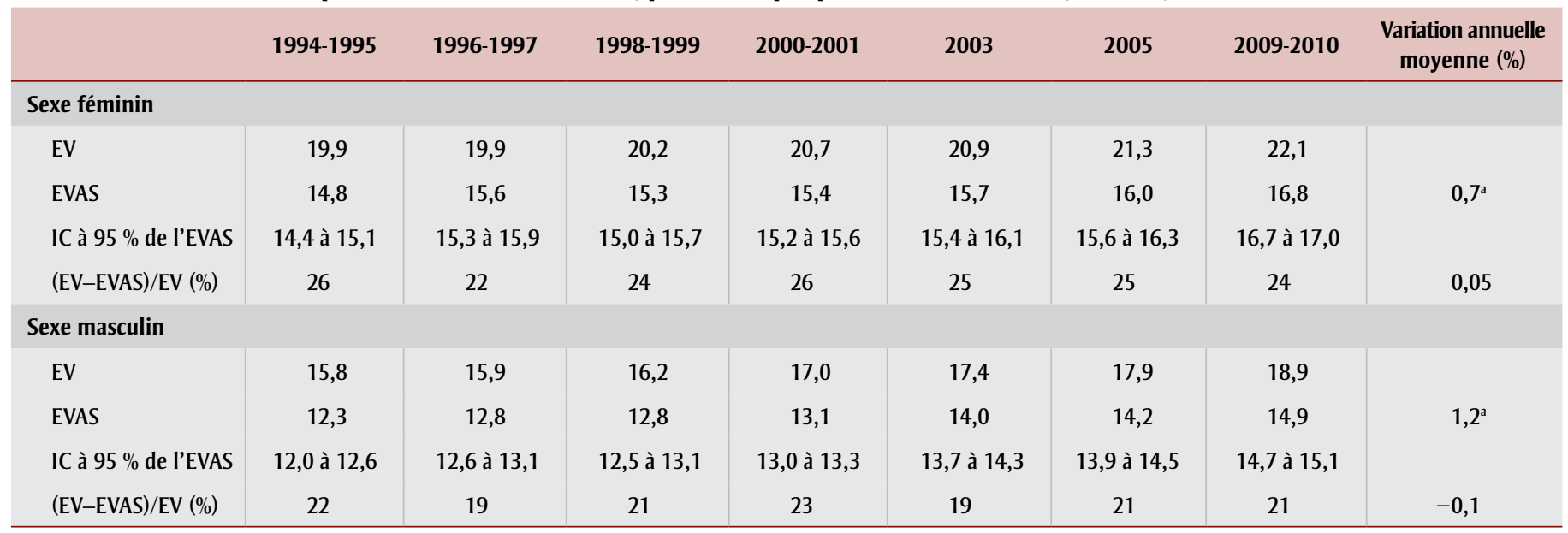

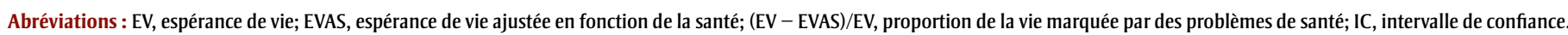
a $p<0,05$.

L'EVAS à 65 ans a augmenté de façon significative dans toutes les provinces sauf à Terre-Neuve-et-Labrador et en NouvelleÉcosse.

Sur le plan de la proportion de la vie marquée par des problèmes de santé, aucun changement notable n’a été observé à la naissance et à 65 ans, dans la majorité des provinces comme à l'échelle nationale, pour la période à l'étude. Terre-Neuveet-Labrador et l'Île-du-Prince-Édouard ont constitué des exceptions : une forte augmentation a été constatée à la naissance, ce qui indique une expansion de la morbidité. Après une stratification des résultats selon le sexe (non présentée), la hausse significative de la proportion de la vie marquée par des problèmes de santé à la naissance n'était plus présente que chez les garçons de Terre-Neuve-et-Labrador et de l'Île-du-Prince-Édouard. Les hommes de Terre-Neuve-et-Labrador ont aussi présenté une hausse significative de cette proportion à 65 ans, tandis qu'une diminution significative a été observée chez ceux du Manitoba.

\section{Analyse}

Notre étude brosse un portrait détaillé des tendances relatives à la compression et à l'expansion de la morbidité au Canada d'après l'évolution de l'espérance de vie (EV) et de l'espérance de vie ajustée en fonction de la santé (EVAS) de 1994 à 2010. Nos résultats montrent que l'EV et l'EVAS ont augmenté chez les deux sexes durant la période à l'étude. Bien que la proportion de la vie marquée par des problèmes de santé ait fluctué, l'augmentation annuelle moyenne non statistiquement significative de cette proportion durant la période à l'étude semble indiquer que, dans l'ensemble, il n'y a eu ni compression ni expansion de la morbidité au Canada entre 1994 et 2010. L'absence de tendance nette est aussi visible dans la répartition des différences sur le

TABLEAU 2

Contribution des composantes mortalité et morbidité aux différences sur le plan de l'EVAS à la naissance, pour chaque période de déclaration par rapport à la période de déclaration initiale, par sexe, Canada, 1994-2010

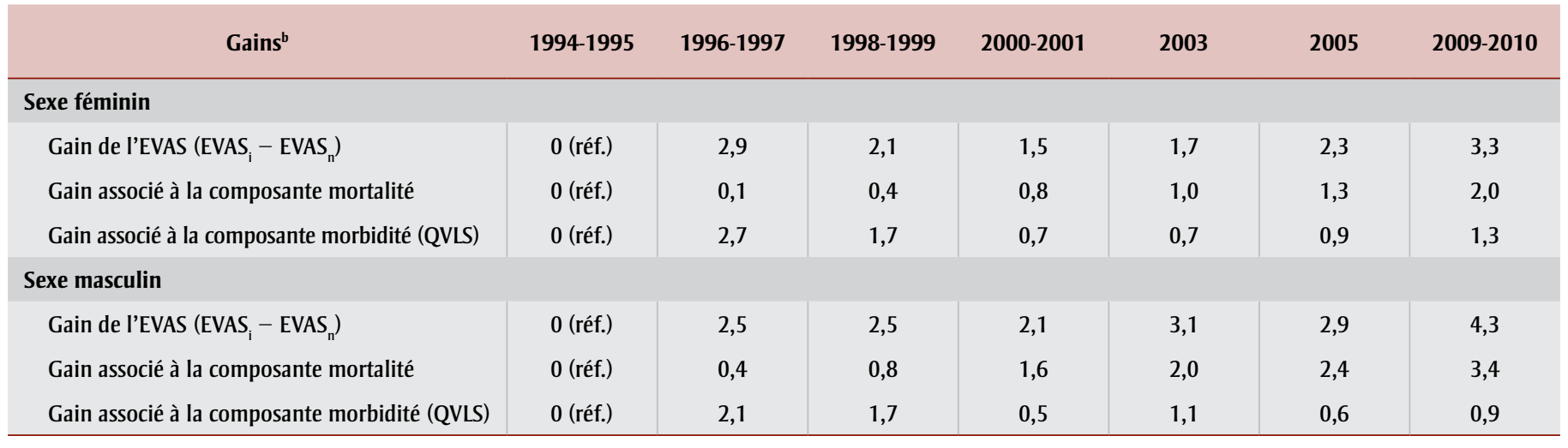

Abréviations : EVAS $_{\mathrm{i}}$, espérance de vie ajustée en fonction de la santé pour la période de référence (1994-1995); EVAS$_{n}$, espérance de vie ajustée en fonction de la santé pour la période n; QVLS, qualité de vie liée à la santé; réf., groupe de référence.

a Il est possible que la somme des gains associés aux composantes mortalité et morbidité ne corresponde pas exactement au gain de l'EVAS en raison de l'arrondissement des chiffres.

${ }^{\mathrm{b}}$ Les gains sont exprimés en années. 
TABLEAU 3A

Variation annuelle moyenne de l'espérance de vie ajustée en fonction de la santé et de la proportion de la vie marquée par des problèmes de santé à la naissance, les deux sexes confondus, par province, Canada, 1994-2010

\begin{tabular}{|c|c|c|c|c|c|c|c|c|}
\hline & 1994-1995 & 1996-1997 & 1998-1999 & 2000-2001 & 2003 & 2005 & 2009-2010 & $\begin{array}{c}\text { Variation annuelle } \\
\text { moyenne }(\%)\end{array}$ \\
\hline \multicolumn{9}{|l|}{ Alberta } \\
\hline$(\mathrm{EV}-\mathrm{EVAS}) / \mathrm{EL}(\%)$ & 13 & 10 & 11 & 14 & 13 & 13 & 13 & $+0,7$ \\
\hline \multicolumn{9}{|c|}{ Colombie-Britannique } \\
\hline \multicolumn{9}{|l|}{ Manitoba } \\
\hline EVAS & 68,0 & 70,4 & 68,8 & 69,3 & 69,7 & 70,2 & 70,0 & $+0,1$ \\
\hline$(\mathrm{EV}-\mathrm{EVAS}) / \mathrm{EL}(\%)$ & 13 & 10 & 12 & 12 & 12 & 12 & 13 & $+0,4$ \\
\hline \multicolumn{9}{|l|}{ Nouveau-Brunswick } \\
\hline \multicolumn{9}{|c|}{ Terre-Neuve-et-Labrador } \\
\hline EVAS & 68,5 & 71,0 & 71,5 & 69,1 & 68,5 & 68,2 & 69,2 & $-0,1$ \\
\hline$(\mathrm{EV}-\mathrm{EVAS}) / \mathrm{EL}(\%)$ & 11 & 8 & 8 & 11 & 12 & 13 & 13 & $+2,5^{\mathrm{a}}$ \\
\hline \multicolumn{9}{|l|}{ Nouvelle-Écosse } \\
\hline EVAS & 65,8 & 68,9 & 69,3 & 68,2 & 68,9 & 68,5 & 69,2 & $+0,2$ \\
\hline$(\mathrm{EV}-\mathrm{EVAS}) / \mathrm{EL}(\%)$ & 15 & 11 & 12 & 14 & 13 & 14 & 14 & $+0,3$ \\
\hline \multicolumn{9}{|l|}{ Ontario } \\
\hline EVAS & 67,7 & 71,4 & 70,7 & 69,5 & 70,4 & 71,0 & 71,7 & $+0,2$ \\
\hline$(\mathrm{EV}-\mathrm{EVAS}) / \mathrm{EL}(\%)$ & 14 & 10 & 11 & 13 & 13 & 12 & 13 & $+0,7$ \\
\hline$(\mathrm{EV}-\mathrm{EVAS}) / \mathrm{EL}(\%)$ & 11 & 9 & 10 & 10 & 10 & 11 & 10 & $+0,4$ \\
\hline \multicolumn{9}{|l|}{ Saskatchewan } \\
\hline EVAS & 68,7 & 70,3 & 70,8 & 69,3 & 70,5 & 69,7 & 70,1 & $+0,0$ \\
\hline$($ EV-EVAS)/EL (\%) & 13 & 11 & 10 & 13 & 11 & 13 & 12 & $+0,4$ \\
\hline
\end{tabular}

Abréviations : EVAS, espérance de vie ajustée en fonction de la santé; (EV - EVAS)/EL, proportion de la vie marquée par des problèmes de santé.

a $p<0,05$.

plan de l'EVAS, qui n'a fait ressortir aucune tendance constante à la hausse ou à la baisse associée à la composante morbidité (QVLS).

Les tendances par province indiquent que, même si les valeurs de l'espérance de la santé traduisent généralement une certaine stabilité entre 1994-1995 et 2009-2010, il pourrait y avoir eu une expansion de la morbidité chez les hommes de Terre-Neuve-et-Labrador et de l'île-du-Prince-Édouard. L'exclusion des données de 1996-1997, cycle caractérisé par une hausse cliniquement importante de l'IES, n'a eu aucune incidence sur les résultats. D’autres études sont nécessaires pour déterminer les déterminants de la santé susceptibles d'être responsables des changements observés à Terre-Neuve-et-Labrador et à l'île-duPrince-Édouard.
Selon une étude antérieure sur les tendances en matière de morbidité chez les adultes canadiens, le rapport entre l'espérance de vie sans incapacité et l'espérance de vie aurait diminué, laissant supposer une expansion de la morbidité entre 1994-1995 et $2007^{19}$. Cependant, comme cette étude était seulement fondée sur deux périodes de déclaration, aucune interprétation valable des variations annuelles survenues durant cette période n'est possible. 
TABLEAU 3B

Variation annuelle moyenne de l'espérance de vie ajustée en fonction de la santé et de la proportion de la vie marquée par des problèmes de santé à 65 ans, les deux sexes confondus, par province, Canada, 1994-2010

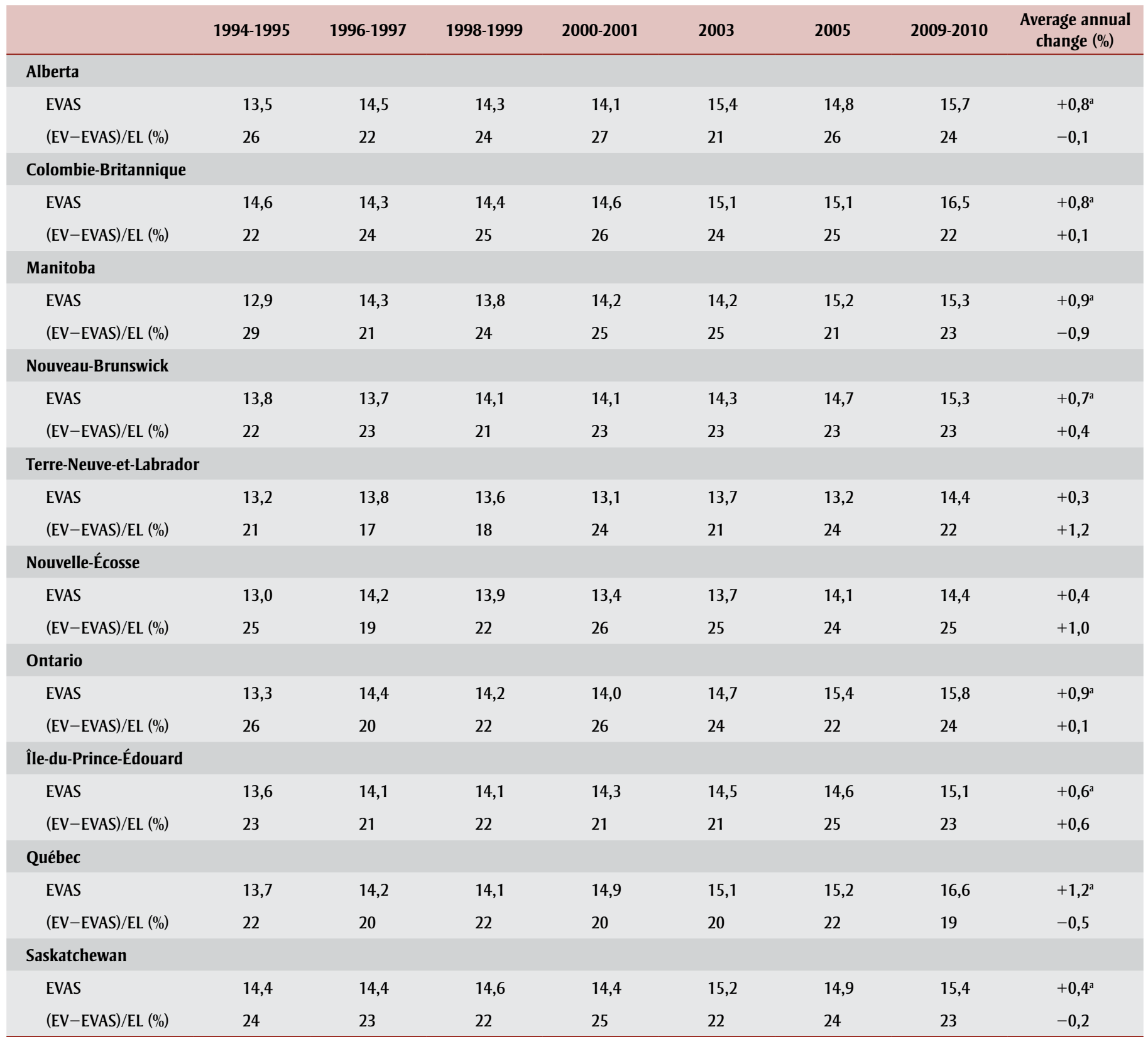

Abréviations : EVAS, espérance de vie ajustée en fonction de la santé; (EV-EVAS)/EL, proportion de la vie marquée par des problèmes de santé.

${ }^{\text {a }} \boldsymbol{p}<\mathbf{0 , 0 5}$.

Nos résultats sont similaires à ceux d'une étude sur les tendances en matière d'incapacité au sein des pays de l'OCDE ${ }^{31}$, selon laquelle la prévalence des incapacités, mesurée par les limites dans les activités de la vie quotidienne, est demeurée stable au sein de la population canadienne de 65 ans et plus entre 1996 et 2003 . En ce qui a trait aux résultats provinciaux, une étude du Québec ${ }^{22}$ a porté sur 1986-1998, une période globalement antérieure à celle visée par notre étude (1994-2010), rendant des comparaisons difficiles à établir. Cette étude a fait état d'une expansion de la morbidité, mais là aussi, l'utilisation de seulement deux périodes de déclaration a empêché toute interprétation valable des tendances.

Nos résultats confortent dans une certaine mesure l'hypothèse de Robine et Michel, ${ }^{32}$ qui ont prédit que les pays économiquement développés présenteraient une expansion initiale de la morbidité en raison de la hausse des taux de survie avec affection incapacitante, suivie d'une compression au fur et à mesure de l'avancement des traitements des maladies chroniques et de l'amélioration des comportements de santé des nouvelles cohortes de personnes âgées. Il est possible que le Canada soit passé de la période d'expansion initiale de la morbidité des années 1980 et 1990 à une 
période de stabilité due à l'avancement de la prévention des maladies chroniques et de la promotion de la santé. Une évaluation des tendances concernant l'espérance de vie en bonne santé au Canada durant cette période antérieure ${ }^{33}$ indique qu'il y a eu une expansion de la morbidité entre 1986 et 1991. Toutefois, pour confirmer cette hypothèse, il faudra suivre ces tendances sur de plus longues périodes. Robine et Michel ont aussi émis l'hypothèse que cette " transition de l'incapacité » comporterait une phase finale durant laquelle il y aurait une nouvelle expansion de la morbidité à la suite de l'émergence de populations très âgées et fragiles ${ }^{32}$. Confirmer cette hypothèse nécessite aussi un suivi régulier au sein de la population canadienne.

\section{Points forts et limites}

Le principal point fort de notre étude a été sa capacité à faire ressortir les modifications de l'espérance de vie en bonne santé à l'échelle de la population sur une période raisonnablement longue, ponctuée de plusieurs points de déclaration. L'étude reposait sur sept points de déclaration représentant 15 années d'observation, tirés de deux grandes études nationales sur la santé ayant utilisé pratiquement le même cadre d'échantillonnage de la population canadienne vivant dans un ménage et la même mesure de qualité de vie liée à la santé. La durée de la période de déclaration et la fréquence des points de déclaration de notre étude sont supérieures à celles de toutes les autres études que nous avons recensées dans la littérature.

Notre étude comportait plusieurs limites. Il n'a pas été possible d'évaluer l'incidence des facteurs socioéconomiques et comportementaux sur l'évolution de l'espérance de vie en bonne santé. Par exemple, l'espérance de vie en bonne santé diminue peut-être dans certaines provinces à la suite de la migration d'adultes en bonne santé vers d'autres provinces pour des raisons liées au travail ${ }^{34}$. De nouvelles études sont nécessaires pour vérifier cette hypothèse, ainsi que pour analyser l'influence d'autres facteurs socioéconomiques possibles. Contrairement à la plupart des autres études sur l'évolution de l'espérance de vie, la nôtre ne comportait aucune mesure des limites dans les activités de la vie quotidienne. Cela restreint notre capacité à établir des comparaisons avec les résultats d'études menées dans d'autres pays. Cependant, une étude de validation de plusieurs mesures de l'incapacité ${ }^{35}$ a révélé que l'IES était bien corrélé avec une mesure des restrictions d'activité et de participation, ce qui laisse penser que les tendances mesurées par l'IES correspondent à celles mises en évidence par les autres mesures.

Il importe de noter que notre étude ne visait pas non plus à évaluer un troisième scénario de morbidité proposé, appelé " équilibre dynamique ${ }^{36}$. Selon ce scénario, à mesure que l'espérance de vie augmente, un état d'équilibre est atteint lorsque la hausse de la proportion de la vie vécue avec des maladies chroniques est contrebalancée par la baisse de la gravité de ces maladies. Les prochaines études sur l'évolution de la morbidité au Canada devraient incorporer des mesures plus explicites du degré d'incapacité afin de déterminer si ce scénario s'applique à la population canadienne.

En ce qui concerne nos sources de données, l'ENSP et l'ESCC excluent toutes deux la population canadienne habitant dans des établissements de soins de santé, en particulier ceux offrant des soins de longue durée. De plus, comme l'ESCC ne vise que la population de 12 ans et plus, nous avons dû appliquer des valeurs hypothétiques de l'IES à la population de moins de 12 ans. Il est possible que les valeurs d'espérance de vie de ces populations exclues diffèrent de celles de la population vivant dans un ménage. Cela pourrait théoriquement affecter l'allure des tendances durant la période de déclaration. Par exemple, comme les personnes âgées sont de plus en plus incitées à rester à domicile, le pourcentage de personnes atteintes d'une incapacité dans la population générale pourrait aller en augmentant.

Les deuxième (1996-1997) et troisième (1998-1999) cycles de l'ENSP portent à la fois sur un échantillon transversal de la population canadienne vivant dans un ménage et sur les membres d'un panel de la cohorte longitudinale de l'enquête. Il est possible que les résultats en matière d'espérance de santé de ces deux cycles diffèrent de ceux des cycles exclusivement transversaux de l'enquête, car les membres du panel survivants pourraient avoir contribué à un effet de "volontaires en bonne santé » : en effet, leur capacité de survivre et de participer aux suivis subséquents pourrait être attribuable au fait qu'ils étaient en meilleure santé que la moyenne de la population ${ }^{37}$. D'ailleurs, nous avons observé que les valeurs moyennes de l'IES étaient élevées pour ces deux cycles. Nous avons effectué une analyse de la sensibilité en excluant de nos calculs les données du cycle de 1996-1997 : les résultats ont confirmé l'existence d'une expansion statistiquement significative de la morbidité chez les garçons nouveau-nés de Terre-Neuve-et-Labrador et de l'Île-duPrince-Édouard, ainsi qu'une stabilité relative de l'ensemble de la population canadienne.

\section{Conclusion}

Notre étude a analysé l'évolution de la morbidité en lien avec l'évolution de l'espérance de vie à la naissance, à 20 ans et à 65 ans. Dans le contexte d'une population vieillissante, il est important de surveiller ces deux indicateurs, car l'expansion et la compression de la morbidité ont des incidences très différentes sur les tendances à venir dans les dépenses en soins de santé. L'absence de données comparables a jusqu'à présent posé problème, mais notre étude fournit des renseignements utiles sur l'évolution de la situation au Canada sur 15 ans. Nos résultats à l'échelle provinciale ont également comblé des lacunes.

Contrairement aux autres pays industrialisés, où des études ont fait ressortir des compressions et des expansions de la morbidité durant les 30 à 40 dernières années, le Canada dans son ensemble semble avoir traversé une période de stabilité relative sur le plan de l'espérance de santé entre 1994 et 2010. Il semble cependant y avoir eu une expansion de la morbidité à Terre-Neuve-et-Labrador et à l'Îledu-Prince-Édouard, particulièrement chez les hommes. Il sera nécessaire de continuer à surveiller l'espérance de santé pour détecter tout autre changement indiquant une hausse de la morbidité. D'autres études devraient être effectuées pour confirmer ces observations et éclaircir les causes de cette expansion de la morbidité à Terre-Neuve-et-Labrador et à l'Île-duPrince-Édouard.

\section{Remerciements}

Nous remercions Heather Orpana, de la Direction générale de la promotion de la santé et de la prévention des maladies chroniques, de l'Agence de la santé publique du Canada, de ses conseils utiles 
quant à l'analyse et aux méthodes employées dans cette étude.

\section{Déclaration sur l'accès aux données}

L'accès aux données ayant servi à cette étude a été octroyé par le biais d'accords de partage conclus avec Statistique Canada et les provinces et territoires canadiens. D'autres chercheurs peuvent avoir accès aux données de Statistique Canada au moyen de son Initiative de démocratisation des données.

\section{Conflits d'intérêts}

Aucun financement n'a été alloué à cette étude. Les auteurs n'ont aucun conflit d'intérêts à déclarer.

\section{Contributions des auteurs}

Colin Steensma a participé à la conception de l'étude, à l'interprétation des données, à la rédaction de l'article ainsi qu'à sa relecture critique. Lidia Loukine a participé à la conception de l'étude, aux analyses statistiques ainsi qu'à la rédaction et à la relecture critique de l'article. Bernard Choi a participé à la conception de l'étude ainsi qu'à la rédaction et la relecture critique de l'article.

\section{Références}

1. Fries JF. Aging, natural death, and the compression of morbidity. Bull World Health Organ. 2002;80(3):245-250.

2. Fries JF. Measuring and monitoring success in compressing morbidity. Ann Intern Med. 2003;139(5 Part 2): 455-459.

3. Fries J, Green L, Levine S. Health promotion and the compression of morbidity. The Lancet 1989;333(8636): 481-483.

4. Gruenberg EM. The failures of success. Milbank Memorial Fund Q/Health Soc. 1977;55(1):3-24.

5. Kramer M. The rising pandemic of mental disorders and associated chronic diseases and disabilities. Acta Psychiatr Scand. 1980;62(S285):382397.
6. Sanders BS. Measuring community health levels. Am J Public Health Nations Health. 1964;54(7):1063-1070.

7. Sullivan DF. A single index of mortality and morbidity. HSMHA Health Rep. 1971;86(4):347-354.

8. Comité directeur sur l'espérance de vie ajustée en fonction de l'état de santé de l'Agence de la santé publique du Canada. L'espérance de vie ajustée en fonction de l'état de santé au Canada : Rapport de 2012 présenté par l'Agence de la santé publique du Canada. Ottawa (Ont) : Agence de la santé publique du Canada; 2012. [HP35-32/2012E]

9. Mathers C. Trends in health expectancies in Australia 1981-1993. J Australian Popul Assoc. 1996;13(1):1-15.

10. Hashimoto S, Kawado M, Seko R, et al. Trends in disability-free life expectancy in Japan, 1995-2004. J Epidemiol. 2010;20(4):308-312.

11. Graham P, Blakely T, Davis P, Sporle A, Pearce N. Compression, expansion, or dynamic equilibrium? The evolution of health expectancy in New Zealand. J Epidemiol Community Health. 2004;58(8):659-666.

12. Cheung KSL, Yip PSF. Trends in healthy life expectancy in Hong Kong SAR 1996-2008. Eur J Ageing. 2010; 7(4):257-269.

13. Doblhammer G, Kytir J. Compression or expansion of morbidity? Trends in healthy-life expectancy in the elderly Austrian population between 1978 and 1998. Soc Sci Med. 2001;52(3):385-391.

14. Bronnum-Hansen H. Health expectancy in Denmark, 1987-2000. Eur J Public Health. 2005;15(1):20-25.

15. Stewart ST, Cutler DM, Rosen AB. US trends in quality-adjusted life expectancy from 1987 to 2008: combining national surveys to more broadly track the health of the nation. Am J Public Health. 2013;103(11):e78-e87.

16. Sagardui-Villamor J, Guallar-Castillón P, Garcia-Ferruelo M, Banegas JR, Rodriguez-Artalejo F. Trends in disability and disability-free life expectancy among elderly people in Spain: 1986-1999. J Gerontol A Biol Sci Med Sci. 2005;60(8):1028-1034.
17. Cambois E, Clavel A, Romieu I, Robine J. Trends in disability-free life expectancy at age 65 in France: consistent and diverging patterns according to the underlying disability measure. Eur J Ageing. 2008;5(4):287-298.

18. Martel L, Bélanger A. Une analyse de l'évolution de l'espérance de vie sans dépendance au Canada entre 1986 et 1996. Dans : Bélanger A, Gilbert S (dir.). Rapport sur l'état de la population du Canada 1998-1999 (p. 171194). Ottawa (Ont.) : Statistique Canada; 1999. [91-209-XIE]

19. Mandich S, Margolis R. Changes in disability-free life expectancy in Canada between 1994 and 2007. Can Studies Popul. 2014;41(1-2):192-208.

20. Jia H, Zack MM, Thompson WW. State quality-adjusted life expectancy for US adults from 1993 to 2008. Qual Life Res. 2011;20(6):853-863.

21. Pinheiro P, Krämer A. Calculation of health expectancies with administrative data for North Rhine-Westphalia, a Federal State of Germany, 1999-2005. Popul Health Metr. 2009;19(7):4.

22. Pampalon R, Choinière $M$, Rochon $M$. L'espérance de santé au Québec. Dans : Camirand J, Aubin J, Audet N, et al. (dir.), Enquête québécoise sur les limitations d'activités 1998. Québec : Institut de la statistique du Québec. 2001;399-420.

23. Tambay J, Catlin G. Plan d'échantillonnage de l'Enquête nationale sur la santé de la population. Rapports sur la santé. 1995;7(1):31-42.

24. Béland Y. Canadian Community Health Survey - methodological overview. Health Rep. 2002;13(3):9-14.

25. Statistique Canada. CANSIM database: Tableau 051-0001 : Estimations de la population, selon le groupe d'âge et le sexe au 1er juillet, Canada, provinces et territoires, annuel (personnes sauf indication contraire) [Internet]. Ottawa (Ont.) : Statistique Canada; [modifié le 28 sept. 2016; consulté le 30 juin 2015]. En ligne à : http://www5 .statcan.gc.ca/cansim/a26?id $=0510001$ \&pattern $=\& \mathrm{p} 2=37 \&$ stByVal $=1 \& \mathrm{p} 1$ $=1 \&$ tabMode $=$ dataTable $\&$ paSer $=$ $\& \operatorname{csid}=$ \&retrLang $=$ fra\&lang $=$ fra 
26. Feeny D, Furlong W, Torrance GW, et al. Multiattribute and single-attribute utility functions for the Health Utilities Index Mark 3 system. Med Care. 2002; 40(2):113-128.

27. Chiang CL. The life table and its applications. Malabar (FL): Robert E. Krieger Publishing; 1984.

28. Hsieh JJ. A general theory of life table construction and a precise abridged life table method. Biom J. 1991;33(2): 143-162.

29. Arriaga EE. Measuring and explaining the change in life expectancies. Demography. 1984;21(1):83-96.

30. Nusselder WJ, Looman CW. Decomposition of differences in health expectancy by cause. Demography. 2004; 41(2):315-334.

31. Lafortune G, Balestat G. Trends in severe disability among elderly people: assessing the evidence in 12 OECD countries and the future implications. Documents de travail de l'OCDE sur la santé $\mathrm{n}^{\circ}$ 26. Paris : OCDE; 2007.

32. Robine JM, Michel JP. Looking forward to a general theory on population aging. J Gerontol A Biol Sci Med Sci. 2004;59(6):M590-597.

33. Chen J, Wilkins R, Ng E. Health expectancy by immigrant status, 1986 and 1991. Health Rep. 1996;8(3):29-38.

34. Norman P, Boyle P, Rees P. Selective migration, health and deprivation: a longitudinal analysis. Soc Sci Med. 2005;60(12):2755-2771.

35. Altman BM, Ng E, Berthelot JM. A comparative analysis of four disability/functional limitation modules in the 2003 Joint Canada/United States Survey of Health. Dans : Proceedings of the 2004 Joint Statistical Meetings of the American Statistical Association. Alexandria (VA) : American Statistical Association; 2004:3256-3263.

36. Manton K, Gu X, Lowrimore GR. Cohort changes in active life expectancy in the U.S. elderly population: experience from the 1982-2004 National Long-Term Care Survey. J Gerontol B Psychol Sci Soc Sci. 2008;63(5):S269S281.
37. Delgado-Rodriguez M, Llorca J. Bias. J Epidemiol Community Health. 2004; 58(8):635-641. 